\title{
Téoros
}

Revue de recherche en tourisme

\section{De Lower St. Lawrence à Charlevoix}

L'émergence d'un haut lieu de villégiature de la bourgeoisie marchande canadienne

\section{Serge Gagnon et Gilles Ritchot}

Volume 17, numéro 1, printemps 1998

Le tourisme dans un Pays à part : Charlevoix

URI : https://id.erudit.org/iderudit/1072371ar

DOI : https://doi.org/10.7202/1072371ar

Aller au sommaire du numéro

Éditeur(s)

Université du Québec à Montréal

ISSN

0712-8657 (imprimé)

1923-2705 (numérique)

Découvrir la revue

Citer cet article

Gagnon, S. \& Ritchot, G. (1998). De Lower St. Lawrence à Charlevoix :

l'émergence d'un haut lieu de villégiature de la bourgeoisie marchande

canadienne. Téoros, 17(1), 15-22. https://doi.org/10.7202/1072371ar d'utilisation que vous pouvez consulter en ligne.

https://apropos.erudit.org/fr/usagers/politique-dutilisation/ 


\title{
De Lower St. LaWrence ।
}

\section{À Charlevoix}

\section{L'ÉMERGENCE D'UN HAUT LIEU DE VILLÉGIATURE DE LA BOURGEOISIE MARCHANDE CANADIENNE}

\author{
Serge Gagnon, étudiant au doctorat \\ Département de géographie, Université Laval, CELAT ${ }^{2}$ \\ Avec la collaboration de Gilles Ritchot, professeur \\ Département de géographie, Université Laval, CELAT ${ }^{2}$
}

"C'est un petit volume qu'il faudrait écrire sur La Malbaie, un perit volume sur papier de soie rose, frais, mêlant l'odeur du varech au parfum de l'heliotrope [... / Rienn'est plus pittoresque, plus rafraichissant, plus varié, phus gracieux que ce morcean de paradis terrestre égaré sur le flanc des Laurentides. Quelle diversité, quelle fécondité, quels luxueux caprices de la nature !

Arthur Butes, (1884: 176).

*La région de Charlevoix est au Québec un cas exceptionnel d'une transition sans heurt entre la villégiature d'hier et celle d'aujourd'hui. On y trouve encore les infrastructures traditionnelles d'une station de vacances, avec son hotel, ses pensions et auberges et ses résidences estivales, et ce, pour le pius grand plaisir du vacancier qui renoue alors tout naturellement avec deux cents ans d'une tradition d'hospitalite, d'un mode de vie né sous le signe du romantismes

Paul Trépanier, (1988: 5).

Cet article se propose de relativiser l'interprétation classique de la spatialisation. de la structuration et de la transformation géohistorique de la région touristique de Charlevoix. Il s'agit de montrer que l'édification d'une villégiature somptuaire à la fin du XIX ${ }^{e}$ siècle, dans Charlevoix, représente l'aboutissement du processus de structuration de cette région touristique. Des motivations autres que socioéconomiques ont contribué à la mise en place de cette région, ainsi qu'à sa valorisation et à sa pérennité jusqu'à aujourd'hui. A la fin du XVIII' siècle, le milieu naturel québécois est convoité par deux acteurs: l'aristocratie terrienne en déclin et la bourgeoisie marchande en ascension. Une lutte s'engage entre ces rivaux, la partie se jouant sur le terrain de l'appropriation territoriale du Saguenay, l'ouver- ture à la colonisation aura été la condition de possibilité d'une vaste opération de tourisme en Charlevoix. L'article montre ainsi que l'activité économique du tourisme est venue rentabiliser une occupation ayant présupposé l'appropriation politique d'un espace qui était déjà investi d'une motivation anthropologique profonde.

\section{LA VISION SANITAIRE DE LA CAMPAGNE}

Au XIX siècle, la manifestation du tourisme dans une région déterminée était souvent associée: d'une part, à des transformations culturelles de la société, notamment l'hygiénisme urbain et l'industrialisation; d'autre part, au développe- ment des moyens de transport, tels les bateaux à vapeur et les chemins de fer qui rendaient accessibles des territoires jusqu'alors peu fréquentés.

L'urbanisation galopante, surtout en Angleterre, provoque des conditions d'insalubrité dans les villes et, parallèlement, une idéalisation de la campagne et de ses vertus sanitaires. Les individus veulent se libérer de leurs angoisses et se réfugient dans la cnature». Celle-ci devient l'antidote aux méfaits de la société industrielle (pollution, saleté, pauvreté, etc.). Au Canada, dans la première moitié du XIX siècle, la population des villes fait face à cette situation. Une arrivée massive d'immigrants irlandais coïncide avec une épidémie de choléra asiatique (1832), qui fait des ravages dans la ville de Québec. Cette conjonction d'événements suscite le départ des bourgeois vers la périphérie. L'attrait de la campagne s'avérant alors irrésistible pour les plus fortunés en quête de lieux paisibles.

*Cewx qui ont les movens quittent aussitót la capitale devenue insalubre pour gagner la campagne environnante. L'épidémie éntraîne à sa suite des changements considerables dans la vie sociale de Québec. Du coup, La Malbaie se métamor. phose en un centre d'attraction qui n'est plus réservé aux amis du cercle intime des seigneurs

(Dube, 1986: 51).

Cette «vision salutaire de la campagnew serait à l'origine de la promotion des destinations touristiques. En effet, au début du XIX ${ }^{\mathrm{c}}$ siècle, avec la médicalisation de la sociéte et l'essor des sciences 
naturelles, se crée un hygiénisme urbain qui intègre cette quête de la nature dans des projets de réforme sanitaire en ville (aqueducs, égouts, éclairage des rues, etc.). Considérant que la ville engendre la mort, la wporte de sortiew est la campagne, promuc comme une anature" empreinte de salubrité. Le corps médical recommandait fortement les diverses stations balnéaires ou thermales, à cause de leurs vertus intrinsèques de guérison associées à la douceur du climat, aux bienfaits de la végétation et des eaux, etc. Mais, selon l'historien du tourisme Marc Boyer, la fonction sanitaire, ou les bienfaits médicaux des stations touristiques,

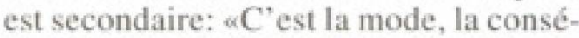
cration par les grands, les rois et princes qui fait la réputation des stations, et non la vertu intrinsèque des eaux* (1980: 53).

Done, l'argument hygiếniste et les vertus sanitaires des stations ont certainement contribué à l'essor des régions touristiques, mais leur consécration et leur pérennité sont-elles fondées essentiellement sur ces facteurs ? Est-ce que le départ des bourgeois de Québec est spontané, contraint, ou les deux à la fois ?

\section{LES INNOVATIONS TECHNOLOGIQUES}

Parallèlement aux spécialistes qui attribuent le tourisme à des transformations culturelles de la société, beaucoup d'experts avancent aussi que le développement rapide de cette pratique est en relation étroite avec les innovations technologiques: l'avènement de la navigation à vapeur sur le Saint-Laurent et l'arrivée du chemin de fer au milieu du $\mathrm{XIX}^{\mathrm{c}}$ siècle. Dans Charlevoix, l'augmentation de fréquentation est favorisée par le développement des transports maritimes. Véritables agences de voyage avant la lettre, les compagnies de navigation font valoir la région, et les bateaux à vapeur appareillent tous les jeudis pour mener régulièrement les voyageurs de Québec à La Malbaic. C'est autour de 1840 que les premières colonies d'estivants s'amènent de Québec. Cette période d'affluence coïncide avec la fin du régime seigneurial (1854) et le début du monopole commercial exercé par la bourgeoisie marchande anglo-saxonne. Pour la région de La Malbaie s'amorce alors une nouvelle ère de prospérité.
"Dès lors, le pays montagneaxn'allait plus jamais être le même. La grande activité commerciale crée par ces nouveaux déplacements va finalement auréler La Malbaie d'une répatation inalterable. L'affluence des visiteurs grandit au rythme des saisons et chacun veut satisfaire son désir de voir et de connaître le "grand flewe", en repassani sur les traces deja anciennesd"un peuple qui s'est laisé apprivoiser par ses rives.

(Dubê, 1986: 57).

Les progrès techniques, reliés à la navigation à vapeur et au transport ferroviaire, ont favorisé la diffusion des touristes. Mais il est arrivé que ces performances ont accompagné le développement touristique et l'ont même suivi. France Gagnon signale que: [.... ] la navigation à vapeur est venue stimuler considérablement un développement déjà amoreé $\%$ (1992: 104). Au milieu du XIX ${ }^{e}$ siècle, la navigation à vapeur a déjà plus de trente années d'existence au Canada. Le premier voyage Montréal-Québec accompli par le vapeur Accommodarion, appartenant à John Molson, remonte à 1809. Donc, le développement des grandes stations touristiques au Canada n'est pas le propre du progres technologique au milieu du XIXe siecle. L'avènement de ces nouveaux moyens de transport terrestre et maritime n'a point créé ni précédé la mise en place des régions touristiques.

Peut-on comprendre le processus d'accession à la notoriété touristique de Charlevoix par les seuls événements liés aux besoins de quitter des conditions d'insalubrité urbaine ou par l'avènement de la navigation à vapeur? Certes, ce sont des facteurs qui accompagnent le développement touristique. Mais doit-on réduire le développement de ce secteur d"activité uniquement a l'expression de facteurs socioéconomiques? Comment comprendre que le tourisme soit apparu en certains endroits et non pas dans d'autres? Et pourquoi les villégiateurs bourgeois se sont-ils installés dans Charlevoix et pas ailleurs? Bien sûr, la région serait attractive à cause de la beauté de ses paysages. Mais comment comprendre qu'à l'intérieur mếme de la région de Charlevoix la pratique touristique, au XIX siècle, se soil développée exclusivement à Pointe-auPic, alors que Baie-Saint-Paul était laissé pour compte? Les paysages y sont pourtant aussi saisissants (Raveneau, 1977: 148; Bureau, 1977: 207).

Partant des conclusions d'un mémoire de maîtrise réalisé en 1996, cet article se propose de relativiser l'interprétation classique de la spatial isation, de la structuration et de la transformation géohistorique de la région touristique de Charlevoix. Il s'agit de montrer que l'édification d'une villegiature somptuaire, a la fin du $X I X{ }^{\circ}$ siècle, représente l'aboutissement d'un processus de structuration régionale. Des motivations autres que soció́conomiques ont contribué à la mise en place de cette région, ainsi qu"à sa valorisation et à sa pérennitế jusqu'à aujourd'hui.

En effet, la manifestation tangible du tourisme est motivée par des significations de nature anthropologique ${ }^{3}$ et elle repose par la suite sur une appropriation territoriale de nature politique ${ }^{4}$. Ainsi, le tourisme, comme activité économique, vient rentabiliser une occupation qui valorise une appropriation politique de l'espace géographique. Ce faisant, il convertit plus fondamentalement une visée anthropologique. On reconnaît le parcours de la géographie structurale (Ritchot, 1985. 1991, 1992; Desmarais, 1995; Desmarais et Ritchot, 1997$)^{5}$. Ce cadre théorique et méthodologique coordonne les prémisses de l'épistémologie morphodynamique", de la sémiotique ${ }^{7}$ et de la théorie de la forme urbaine (T.F.U.) , ainsi que le patrimoine empirique et systémique qu'offre la géographie historique (Courville ef al. 1995). Ces approches permettent de développer I'hypothese relativement à la mise en place de la structuration spatiale du tourisme en Charlevoix et d'en reconstituer la dynamique des formes et des forces à l'échelle régionale.

La reconnaissance de Charlevoix conme destination touristique de premier choix aux $X I X^{c}$ et $X X^{t}$ siècles n'a été rendue possible qu'aprês une âpre lutte géopolitique. A la fin du XVIII ${ }^{e}$ siècle, le milieu naturel québécois est convoité par deux acteurs: l'aristocratie terrienne en déclin et la bourgeoisie marchande en ascension. Une lutte s'engage entre ces rivaux, la partic se jouant sur le terrain de l'appropriation territoriale du Saguenay, l'ouverture à la colonisation par les acteurs bourgeois a été la condition de possibilitê d'une vaste opération de tourisme en Charlevoix. L'émergence de 
l'activité touristique dans la vallée du Saint-Laurent était liée à lạ possibilité de la mise en valeur de Charlevoix. Cette valorisation était bloquée, jusqu au milieu du XIXe siecle, par une appropriation de type seigneurial.

Dans ce contexte, la nature a eté investie de valeurs imparties au courant wromantique ${ }^{5}$. L'espace charlevoisien a acquis, en fonction de cette conversion, ses premières lettres de noblesse qu' elle conserverait d'ailleurs encore aujourd' hui (S. Gagnon, 1996: 139-141). Au XIX" sieccle, l'espace touristique québecois était polarisế par le développement de grandes villes, lesquelles ont transposé les valeurs du libéralisme économique de la bourgeoisie industrielle (travail, production, épargne) dans l'amenagement des grands fronts de villégiature (Dubé, 1983, 1985. 1986). Ce tourisme de distinction magnifrait la wnatures (jardins et villas) et communiquait avec panache les valeurs identitaires nationales canadiennes (GagnonPratte, 1980, 1988; Dubé, 1995). L'activité économique du tourisme est donc venue rentabiliser une occupation ayant presuppose l'appropriation politique d'un espace qui était deja investi d'une motivation anthropologique profonde.

En reconduisant le système seigneurial en 1774, le droit colonial a entratine une conséquence qui ne cessera de marquer l'enclave laurentienne, Le nouvel ordre constitutionnel epargnait les titulaires français mais seulement là où les seigneuries étaient en exploitation. Les fiefs qui n'étaient pas vraiment exploités ou les domaines plus ou moins négligés étaient attribues à des seigneurs anglophones. Des fiefs anglophones ont vu le jour avant même que la cession soit confirmée. Les bénéficiaires étaient pour la plupart des militaires hauts-grades qui avaient servi dans les bataillons de l'armée anglaise au moment de la conquête. Au cours des decennies qui suivront, des aristocrates anglophones, militaires pour une bonne part, vont occuper des seigneuries.

\section{LA NAISSANCE DES FRONTS DE VILLÉGIATURE: LA SEIGNEURIE DE LA MALBAIE}

La seigneurie de La Malbaie constituait, depuis 1724 (trente-cing ans avant la Conquête), le centre d'approvisionnement des postes de traite du adomaine du Roy (Gauthier, 1989: 49; Des Gagniers 1994: 148). Cette seigneurie faisait partie intégrante du domaine foncier de la couronne winterdit à la colonisation\%. En $1750,1 \mathrm{c}$ père jésuite Claude-Godefroy Coquart, qualifiait Ia ferme de La Malbaie comme étant al'une des plus belles de tout le Canadas (Gauthicr, 1989: 49). Au sujet de la concession des seigneuries et des valeurs aristocratiques, Fernand Ouellet ecrit:

- Si les valears aristocratiques conservent après 1760 toute leur capa= cite de rayonnement, c'est que la noblesse s'est trouve appuye et renforcé par la présence d'une arixtocratie anglophone. En effet, parmi ceax qui achetent des selgnewries au lendemain de la Conquête, les militaires et les aristocrates anglophones occupent le premier rang et de loin $\times$ (1978: 211).

Dẻs le début du Régime britannique, la seigneurie de La Malbaie est divisée et concédée à deux militaires écossais (figure-Seigneuries). Au sujet du changement decoulant de la Conquête, MarcAndré Bluteau et Serge Gauthier spéci= fient:

- La vie dans la region de BaieSaint-Paul n'a pas veriablement changé. Nen wa tout autrement pour celle de La Malbaie qui, partagee en dew en 1762 T.. / entre les nouveaux seigneurs cossais Nairne ef Fraser, accueille de plas en plas de familles anglophones sur son territoires (1987: 5 ).

Philippe Dubé fait cette remarque ả propos du nouveau dynamisme insufflé à cette région par la Conquête:

«... J La seignearie de la Malbaye, qui a conna ane croisance lente à ses debut, se trouve tout à coup favorise par la conjoncture d'apres-guerre qui amenera l'implantation de nouveaux seigneurs, venus ceux-là d'Écosse (1986: 23).

A l'est de la riviere Malbaie se trouve la seigneurie de Mount-Murray, proprićté de Malcom Fraser et, à l'ouest de cette même rivière, la seigneurie de Murray Bay, propriété de John Nairne. Les deux officiers écossais du $78^{\text {th }}$ Fraser Highlanders obtiennent ces terres dụ général Murray: גes paysages $[. .$.$] ne sont pas sans évo-$ quer ceux de leur Écosse lointaines (Des Gagniers, 1994: 165). Très tôt, la force d'attraction des paysages de la région avait produit son effet aupres des nouveaux arrivants. Le dernier tiers du XVII ${ }^{e}$ siècle amène son cortège de visiteurs (hauts fonctionnaires, gouverneurs, militaires). Le courant romantique déferle sur Murray Bay. Issus de l'aristocratie britannique, un groupe restreint de visiteurs décrivent le paysage comme étant d'une beauté d'une attirance sans pareilles. Ce sont ces premiers élans de sympathie qui attiseront le désir', chez les nouveaux acteurs bourgeois, de fréquenter ce coin de pays.

Voilà pour l'aristocratie britannique. Qu'en était-il de la noblesse canadienne-française qui éprouvait le même engouement pour la nature ? Cette noblesse s'est installée sur la rive sud, à Kamouraska.

\section{LE FRONT DE VILLÉGIATURE DE KAMOURASKA}

Kamouraska fut le premier centre de villégiature au Canada où les membres de I'ellite aristocratique se rencontraient. Joseph Bouchette, lors de sa tournée d"inspection en 1813. l'identifie déjà comme tel (Paradis, 1948: 168), Jean-Pierre SaintAmour note, à l'égard de la clientèle qui fréquente la station de Kamouraska: $\propto \mathrm{Ce}$ fut d'abord à Kamouraska où se rencontrèrent les familles aisées canadiennesfrançaises du début du dix-neuvième siècle. Il semble que ce fut un des seuls centres fréquentés par les francophones [avant 1850]\% (1979: 19), Roger Brière Écrit à ce sujet:

- Le premier sejour d'été auquel se donmerent rendez-vous les fomblles canadiennes-françaises joussant de quelque fortune, fut Kamouraska. Ce village est le seul rendez-vous estival dont nous ayons trowe mention avant l'age des bateaux à vapew et du chemin de fers (1967: 86-87; 1967a: 17).

Un peu plus loin, l'auteur ajoute:

"Nulle part cependant, les villegiateurs n'taient en nombre sabisant pour qu'on pât parler de sta. tion de villegiature au sens actuel. Kamouraska semble avoir ete la 


\section{L'APPROPRIATION BOURGEOISE DU CORRIDOR DU SAGUENAY}
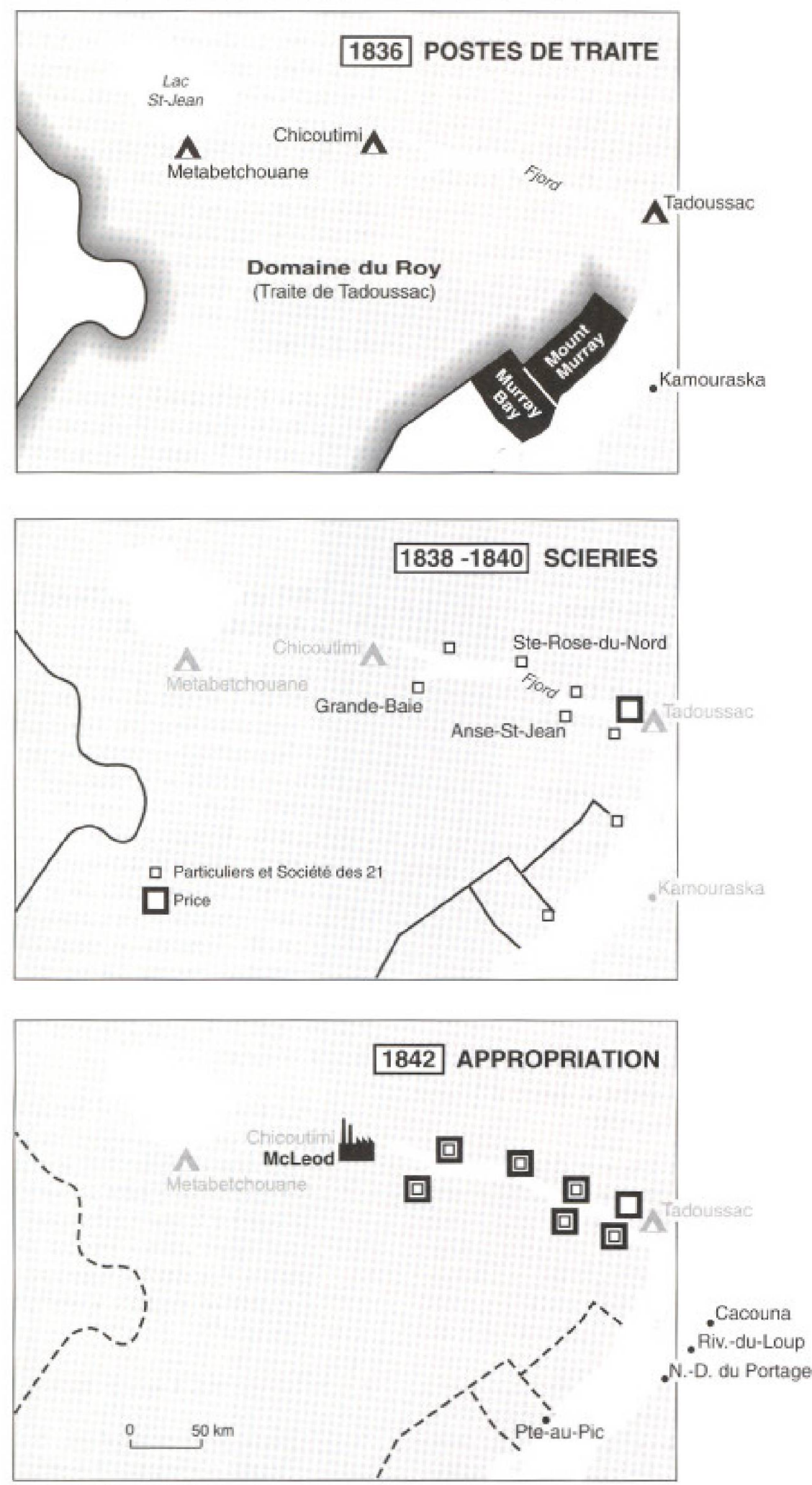

Source. S. Gagnon 1996 : chap. 3. seule exception, dès les premières années da siécle. Ce village fut en effet le premier du Québec à recevoir des citadins en vacances. Sa célébritét datait de l'époque des seigneurs Taché et des fêtes resplendissantes qu'il y domnait [... / On s'y rendait de Montréal et de Québec bien avant que des places d'eau comme Pointe-au-Pic et Cacouna ne fussent connues. Arthur Buies a pu dire que "les autres endroits ne comptaient pas" et que le manoir des seigneurs Taché "avait reçu pendant un quart de siècle tout ce que le pays renfermait d'hommes eminents dans la vie publique ou distingues par la naissance et la position". Pour James Lemoine. "before the area of steamers, in fact even as late as 1850, Kamouraska was the Brighton of Lower Canada" "s (1967: 88; 1967a: 22-23).

Pascal Taché a siégé pendant deux ans à la Chambre d'Assemblée du Bas-Canada (1798-1800). Dès le début du XIXe siècle, il se dévouait à sa seigneurie où il accueillait l'élite aristocratique canadiennefrançaise de l'époque. L'élite se réunissait pour discuter de politique et de stratégie. Elle sentait venir le vent de la bourgeoisie et devait trouver de nouveaux défis pour mobiliser la population canadiennefrançaise. D'où le projet de colonisation du Saguenay.

Contrôlé, tout comme Murray Bay, par les acteurs de l'aristocratie. le front de villégiature de Kamouraska semble avoir été l'endroit où l'on a planifié l'appropriation du Saguenay. Le seigneur Pascal Taché fut l'un des initiateurs des pressions pour ouvrir le Saguenay à la colonisation. Il a ouvré de nombreuses années sur les terres du *domaine du Roy avant de s'établir définitivement dans sa seigneurie (Larocque, 1993: 61). Le seigneur de Kamouraska était fidèle à la Couronne britannique et aux principes du système seigneurial. Comme nous allons le voir, l'aristocratic aurait fait la promotion d'une colonisation agricole dans le but de reconduire à distance le système seigneurial et ses prescriptions hors de la vallée du SaintLaurent.

Au cours de la première moitié du XIX siècle, Kamouraska était donc le rendezvous estival du gratin de la société canadienne-française de l'époque. Murray Bay, 
sur la rive nord, joualt le même rôle pour l'élite britannique. Les salons de Kamouraska ont certainement dû̉ entendre parler de l'exploration du Saguenay. C'était la première tentative de l'aristocratie pour prendre le contrôle de la mobilité des Canadiens-français. L'enjeu de cette dynamiqué géopolitique visait ultimement le contrôle de la vallée du Saint-Laurent. La lutte pour le contrôle du territoire va débuter. Elle va confronter deux acteurs: $I^{+}$aristocratie terrienne installée dans la vallée laurentienne et la bourgeoisic marchande des villes.

\section{L'APPROPRIATION TERRITORIALE DU SAGUENAY}

L'appropriation bourgeoise débuta dans la région du Saguenay, depuis le fond de la baie des $\mathrm{Ha}$ ! Ha ! jusqu'à la décharge du lac Saint-Jean, en passant par le portage de Kénogami, les postes de traite de Tadoussac, Chicoutimi et Metabetchouane (Pointe-Bleue) Cette région se trouvait tout entière incluse dans le domaine de traite de Tadoussac le $_{\text {le }}$ domaine du Roy» (figure - Saguenay).

L'aristocratie avait déjà prévu affecter cette région à une occupation agricole. Le seigneur de Kamouraska voulut qu'une enclave seigneuriale dédoublât, en plein bouclier, la région organisatrice des basses terres laurentiennes. Le projet fut bloqué par le gouverneur en 1829 (G. Gagnon, 1988: 69). De son côté, la bourgeoisic marchande avait essayé de s'emparer des seigneuries laurentiennes. Les seigneurs ecclésiastiques et francophones, qui représentaient la moitié de l'aristocratie terrienne en $1790, s^{\prime}$ accrochèrent à leurs fiefs et en investirent les rentes dans la formation d'une petite bourgeoisie provinciale. En résumé, l'aristocratie et la bourgeoisie étaient frustrées toutes les deux, la première en dehors des seigneuries du Saint-Laurent et la seconde à l'intérieur. Une lutte s'engagea entre ces classes, dont l'enjeu était l'appropriation de l' «élément territorial».

L'aristocratie ayant échoué avec son plan de colonisation agricole du Saguenay, la bourgeoisie marchande pouvait rappliquer. Cet acteur voulait concevoir à sa maniere un établissement neuf, sauf qu"il s"agirait d'un front pionnier agro-forestier éventuellement soumis à la tenure soccagère (propriété privée), En marge de représentations auprès du gouvemement impérial accompagnées de pétitions, de commissions d'enquête et d'une reconnaissance des lieux, une occupation xillégales de l'anse des $\mathrm{Ha}$ ! Ha ! était accomplie en 1836. Les notables de Kamouraska, les aristocrates anglophones de La Malbaic et la compagnie de la Baie d'Hudson contrôlaient toujouts la situation. Mais une brèche avait été pratiquée dans la réserve fonciêre de la Couronne. D'après Serge Courville et Normand Séguin (1996), il faudra près d'un siecle pour que le «squattering" disparaisse completement du canton de Laterrière au sud de Chicou= timi.

A l'approche des troubles de 1837, la métropole britannique veut calmer les esprits. Elle voit d'un bon wil que des particulier's canadiens-français puissent participer aux activités économiques. Elle continue d'appuyer la compagnic de la Baie d'Hudson dans le but d'empêcher les grands marchands de coloniser le Saguenay. Mais les deux acteurs — le gouvernement de Londres et la compagnie royale - s'entendent pour que des entrepreneurs francophones aient la permission d'exploiter la forêt dans le domaine de Tadoussac (Lapointe et al., 1981). Deux entrepreneurs canadiens-français de La Malbaie sautent sur l'occasion. Ils sont de mèche avec l'homme d'affaites William Price (Lalancette, 1987: 9). Une opartie d'échecs» va se dérouler dans le corridor du Saguenay, que fréquentait le seigneur de Kamouraska avant que William Price $y$ avance ses wcavaliers

Les entrepreneurs canadiens-français de La Malbaie ne devaient pas commercer avec les Amérindiens ni autoriser l'agriculture, en même temps qu'ils devaient fournir une caution à la compagnie de la Baie d'Hudson en plus de s'incorporer. Cette dernière obligation donnait lieu, en 1837 , a la fondation de la Société des Vingt-et-un. Son principal actionnaire, Alexis Tremblay-Picoté, était un agent de la compagnie de William Price. Des scieries ont été implantées de proche en proche, depuis les confins des fiefs de La Malbaie jusqu'à la baic des $\mathrm{Ha}$ ! $\mathrm{Ha}$ ! Avec trois postes peuplés d'Amérindiens et de missionnaires, ces usines vont attirer quelques travailleurs en principe tenus de ne pas se prévaloir des défrichements pour ajouter l'agriculture à l'activitế forestière.
Solidement implanté au Saguenay et prêt à recevoir les colons, William Price attend son heure de gloire. Le 2 octobre 1842 , au moment du renouvellement du bail d'affermage du «omaine du Roys à la compagnic de la Baie d'Hudson, le gouvernement central de Londres cède devant la nouvelle situation engendrée par ces mouvements illegaux d'appropriation territoriale (Dechêne, 1977: 706). Les autorités lèvent l'interdit d'établissement permanent sur le territoire du adomaine du Roym, supprimant ainsi la réserve foncière de la couronne (Lalancette, 1987: 11). Désormais, les jour's de l'aristocratie terrienne et du régime seigneurial sont comptés.

A l'invitation des dirigeants de la Société des Vingt-et-un, les colons quittent les seigneuries de Charlevoix (Mount-Murray, Murray Bay, Éboulements, Gouffre et Beaupré) et affluent sur les nouvelles terres exemptes de tenure seigneuriale. Gérard Bouchard caractérise ainsi l'importance du phénomène:

* L'amplear da mouvement d'immigration originant de toutes les parties de Charlevoix, les motifs qui ont poussé les familles à quitter, les conditions dans lesquelles elles liont fait et les témoignages indirects qui en sont restés font plutôt croire à un mouvement presque généralisé [...]. (1989: 395).

La Société des Vingt-et-un avait dû emprunter pour lancer ses chantiers à proximité de La Malbaie, le long du fjord (Anse Saint-Jean, Sainte-Rose-du-Nord, etc.) et dans le fond du havre naturel en eau profonde de la baie des $\mathrm{Ha}$ ! Ha! (Port-Alfred, aujourd' hui la localité de La Baic). Comme elle avait placé en point de mire un plein contrôle de la situation, elle n'hésitait pas à recourir au crédit, au point qu'elle fut incapable de rembourser ses emprunts. En 1843, le créancier en sousmain, nul autre que William Price, utilise le stachats - une saisie à vrai dire-pour récupérer les équipements et, indirectement, prendre pied dans le corridor du Saguenay (G. Gagnon, 1988: 74; Lalancette, 1987: 10). De cette façon, il acquiert les equipements manufacturiers de McLoed à Chicoutimi.

A travers la personne de William Price, c'était la future bourgeoisie marchande qui, forte d'une trajectoire ${ }^{10}$ attractive 
ayant manipulé le nomade ${ }^{11}$ canadienfrançais, s'appropriait le Saguenay aux dépens de l'aristocratie: d'abord la compagnie royale de la Baie d'Hudson; ensuite les seigneurs anglophones de La Malbaie; enfin les notables de Kamouraska. Selon Louise Dechêne, la manouvre permit le contrôle des positions de l'ensemble de I'Est du Québec.

"A lui seul, I'éparpillement du capital et du travail semble indiquer que toute cette entreprise a été, non seulement encouragée, mais créée par Price pour lai permettre de s'installer sur les terres de la Couronne. Le gouvernement et la compagnie de la Baie d'Hudson céderont devant un fait de peuplement, mais auraient-ils aussi facilement lasse un marchand monopoliser whe si vaste région ? (1977: 706).

Il est intéressant de constater que «le North America, un bateau à vapeur [de la com= pagnie "Richelieu \& Ontario"], aurait été le premier navire à se rendre à Chicoutimi en 1842. Pour cette première expérience, trois cents personnes avaient fait des réservations\% (Simard, 1987: 282). Le tourisme a atteint le Saguenay avant la vague massive de colonisation intérieure !

\section{LA CHUTE D'UN POUVOIR ET L'INVESTISSEMENT DU TERRITOIRE CHARLEVOISIEN}

La perspicacité de William Price semble avoir plané au-dessus du moindre doute. Ce marchand comprit que les troubles de 1837 racontaient autre chose qu'un affrontement interethnique, à savoir un rapport de forces politiques. Au lieu de mobiliser des sedentaires anglophones contre l'aristocrate francophone dans la vallée de la survivance, le joueur d'échees attaqua l'aristocrate anglophone dans ses terres de la couronne en mobilisant les nomades francophones de Charlevoix. La victoire de William Price sur le Saguenay consacre le pouvoir de la bourgeoisie marchande dans la vallée du Saint-Laurent.

*A Québec et à Montréal, une nouvelle bourgeoisie vient se greffer $a$ la classe dirigeante de miliaires et administrateurs britanniques du gouvernement colonial. Quelques Canadiens parviennent ainsi d se hisser aux rangs les plus elevés de la hierarchie sociale» (Dubé, 1986: 145).

Le gouvernement décrète, en 1854, l'abolition du régime seigneurial et sanctionne ainsi la mainmise du capitalisme marchand sur l'élite foncière aussi bien anglophone que francophone (Courville et al, 1988: 8). Nous pouvons déjà appeler par leur nom quelques représentants d'une bourgeoisie marchande qui était encore largement américaine et britannique. William Price, le plus influent des aLumber Lords\%, disposail d'un kempires en 1850. Dans la foulée des magnats de Montreal - des McGill, McTavish, Molson et d'autres - il compta parmi les premiers bourgeois canadiens.

Désormais, la bourgeoisie peut librement investir les places naguère détenues par les seigneurs et mettre en valeur la vallec du Saint-Laurent. Une bourgeoisie spécifiquement pancanadienne pouvait enfin contrôler certaines positions-symboles: la proue du cap aux Diamants, la rue SaintJacques et les glacis du mont Royal, NotreDame-du-Portage et Cacouna versus Kamouraska.

Un des sites privilegiés par la nouvelle bourgeoisie sera Charlevoix, ce bastion de la défunte Société des Vingt-et-un. Le front de villégiature sera développé par le grand capital à compter de 1853 , avec la construction par le gouvernement d'un quai à Pointe-au-Pic. L'activité commerciale sur le Saint-Laurent, concentrée entre les mains du grand capital, attire une élite entreprenante et désireuse de mettre en valeur ces régions jusqu'alors difficiles d'accès en raison de l'emprise de la tenure seigneuriale. Dès lors, nous comprenons que la bourgeoisic se devait d'affirmer cette victoire à travers un processus de rentabilisation et d'occupation du territoire approprié. Ce fleuron de la villégiature de luxe sera financé par le grand capital et monumentalisé par une édification somptuaire de grande renommee: des villas sur le boulevard des Falaises et des hôtels, dont le Manoir Richelieu.

\section{CHARLEVOIX: UNE CHASSE GARDÉE}

La mise en scène particulière du centre de villégiature de Pointe-au-Pic et l'effort d'appropriation territoriale déployé par la bourgeoisie pour acquérir cet espace suggèrent que celui-ci aurait été volontairement isolé, à la différence des autres stations touristiques à la mode au XIX siècle (Cacouna, Métis-sur-Mer, etc.). Pourquoi le seul moyen de transport pour atteindre Charlevoix à cette époque, et ce jusqu'en 1919, est-il le bateau à vapeur? Les autres stations étaient pourtant accessibles par train ou par route. L'isolement tardif de Charlevoix fait problème. Le nonaccès à Charlevoix, ni par route ni par chemin de fer, n'a pas traduit la contrainte d'un obstacle naturel mais l'administration d'un ainterditw (Des Gagniers, 1994: 357; Vézina, 1977: 307).

L'isolement de Charlevoix ne peut pas être impute aux seules contraintes topographiques. Comment expliquerait-on alors le fait qu à la même époque le train traverse le Canada d'un océan à l'autre en passant à trawers les Rocheuses? Plus près de Québec, comment expliquerait-on le fait qu'une ligne de chemin de fer atteint Roberval autour de 1880 , traversant à cette fin trois cents kilomètres de forêts et de marécages dans le massif laurentien, alors que Pointe-au-Pic n'est qu'à cent cinquante kilomètres à l'est de Québec?

Une étude exhaustive menée par France Gagnon, sur l'evolution des circuits touristiques au $X X^{s}$ siècle en relation avec les moyens de transport, soulève des interrogations quant à la desserte ferroviaire des stations touristiques et à la possibilité d'isolement de celles qui étaient situées en aval de Québec (1992: 121-127). Son analyse montre que I'implantation progressive du chemin de fer, à partir de la seconde moitié du XIX siècle, a couvert, au tournant du même siècle, la presque totalité de l'écoumène du territoire bas-canadien, à l'exception des régions de Charlevoix et du Bas-Saguenay. Ces dernières ne sont accessibles que par bateau tout au long de cette période. Dans cette optique, aurait-on volontairement isolé Charlevoix dans le but de constituer un domaine réservé à un nouvel acteur dominant: la bourgeoisie ?

Il appert que, dans le but de consacrer ostensiblement la région de Charlevoix à la nouvelle elite bourgeoise, celle-ci l'a constituée en une réserve. Elle a maintenu l'isolement des lieux en bloquant leur accès. Afin de valotiser sa position d'évasion, la grande bourgeoisie aurait contrồlé la position des autres villégiateurs. D'une 
part, ceux-ci étaient dirigés vers d'autres stations touristiques (Cacouna, Métis-surMer, Kamouraska, etc.) desservies par le chemin de fer ou par la route. D'autre part, ils devaient se soumettre à des conditions d'accès particulières pour se rendre a Pointe-au-Pic. Ces conditions seraient représentées par les abateaux blancsw.

L'aceès au centre de villegiature de Charlevoix s'est effectué, jusqu'en 1919, essentiellement par bateaux à vapeur. Comme il a été spécifié précédemment, c est la mise en place du quai par les autorités impériales, en 1853, qui permet d'établir un service régulier de vapeurs entre Québee et Pointe-au-Pic pendant la saison estivale. Les compagnies de navigation, particulièrement la «Richelieu $\&$ Ontarios, façonneront ale développement de la villégiature dans Charlevoix w et vont faire valoir la région en en magnifiant l'accès. En effet, cet accès est comme ritualisé, en fonction d'un franchissement de limites entre les points de départ et d'arrivée. D'un monde posé (Québec), on accéderait à un monde inversé (Pointe-auPic), via le trajet maritime entre ces deux points. Les voyageurs expérimentaient ainsi un «dépaysement $\%$, une atransformations.

Les abateaux blanes représentaient sûrement plus qu'un simple moyen de transport. Ces vapeurs étaient «de véritables palais flottants qui assurent à la fois vitesse, luxe et confort fastueux* (Dubé, 1986: 77). Par leurs apparats, ces navires étaient le véhicule d'une transformation que devaient vivre les villégiateurs pour se conjoindre avec un nouveau monde, un espace diamétralement opposé à celui qu'ils venaient de quitter.

¿Parallèlement. la période des vacances nécessite aussi un changement de lieu, un dépaysement, ce qui crée alors un mouvement inhabituel de la ville à la campagne. Le déplacement de ces citadins vers les contrées éloignées des pavés et du browhaha des foules donne naissance à un nouveau mode de vie: la villégiatures (Dubé, 1986: 103).

Le tourisme de adistinctions va perdurer jusqu'aux années 1960. Les derniers wbateaux blancs circulent en 1965 . Malgré le désinvestissement subi dans la région au cour's des années 1970 , le tourisme installé pendant les années 1980 veut recréer l'atmosphère d'antan, notamment avec l'instauration d'une aubergeric de luxe, le recyclage du Manoir Richelieu et l'ouverture du casino. Désormais cependant, c'est la nouvelle classe d'affaires québécoise qui contrôle la position. Convoquée par l'État - le premier sommet socioéconomique s'est tenu au manoir Richelieu en 1977 - cette nouvelle classe d'affaires transpose les valeurs du néolibéralisme (formation, développement durable, assainissement des finances) dans l'aménagement de nouveaux fronts de villégiature. Ce tourisme wdurablew magnifie la wnature - les baleines - ainsi que la «cultures - les galeries d'arts...

Toutes activités touristiques étant similaires en surface, Charlevoix est un cas exemplaire du fait que sa position repose sur une appropriation spatiale particulière qui lui confere une signification politicoculturelle et anthropologique précise. Étant donné la manière dont la bourgeoisie marchande au $X I X{ }^{e}$ siècle et la nouvelle classe d'affaires au $X^{e}$ siecle se sont approprié ce lieu élu, la position de Charlevoix a acquis un caractère politicoculturel et anthropologique singulier.

Un comité de lecture a lu et accepté ce texte

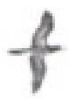

\section{NOTES}

I Dans les guides touristiques au XIXe siècle, la région de Charlevoix fait partie d'un grand ensemble touristique que l'on dénomme *Lower St.Laurences. Ce domaine incluait aussi le Bas-Saguenay, la Côte-du-Sud et le Bas-Saint-Laurent.

2 Centre d'études interdisciplinaires sur les lettres, les arts et les traditions des francophones en Amérique du Nord.

3 Employé comme adjectif et ne renvoyant pas à la science humaine qu' est l'anthropologie, I"anthropologique qualifie un sens, le sens de la vie, des destins individuels et collectifs. Ces représentations $s^{\prime}$ actualisent en espace sous l'effet de l'appropriation. En général, less expressions esens anthropologique ou wvaleur anthropologiques s'emploient au singulier, à la différence des expressions areprécentations symboliquesm ou areprésentations (valeurs) culturellesw. qui soni synonymes à toutes fins pratiques et qui s'cmploient au pluriel. Les définitions géographiques structurales, dans ce texte, sont empruntées à des wo Notes et document de cours» du Département de Géographie de I'Université Laval (GGR-15195).
4 Le politiqué concerne le contrôle de la mobilité par I'appropriation. Par ailleurs, le contrôle de la mobilité se traduit en trajectoires qui engendrent des domaines dont la valorisation par la rente stimule une edification ultérieurement ciblé par la rentabilisation productive ( $c$ f . Ritchot et Mercier: 1992).

5 La theorie gégraphique structurale permet de ramener la diversité des formes d'éablissement à leur unité d'organisation sousjacente (cf. Desmarais, $1995: 37-42)$.

6 La théorie morphodynamiques, élaborée par Renê Thom et développée par Jean Petitot, permet de concevoir, à l'aide d'outils mathematiques, la genese des structures morphologiques (idem : 34-37). La morphologie est l"etude des formes, des structures, On entend généralement par structure : aun tout formé de phénomènes solidaires tels que chacun dépend des autres et ne peut être ce qu'il est que dans et par sal relation avec eux. (Lalande, Vocabulaire de la philosophie, 1972 : 1031). L'Etude de la genthe des formes cherche il savoir pourquoi et comment les structures cmergent et se developpent.

7 La mtherie semiotiques d'Algirdas Julien Greimas permet de spatialiser des contenus provenant de waleurs profondesw véhiculếes par les sociétés à travers un processus géneratif de la signification (c). Desmarais, 1995; 42-46 et 82-91).

8 La théorie géographique structurale de la forme urbaine élaborée a partir de 1976 par Gilles Ritchot et systematisce par Gaetan Desmarais dans un chapitre de son ouvrage sur la Morphogenèse de Paris (1995: 49 . 103). spécific que of l'espace géngraphique est organise par une structure morphologique permettant de faire tenir ensemble la diversité des phénomènes. Ainsi approché, l'établissement des individus et des sociétes à la surface de la terre est organisé par une worme $w_{\text {, }}$ une wstructure de positionsw abs. trailes qui médiatise la transformation des espaces naturels en espaces culturels. (Desmarais et Ritchot : 1997: 44).

9 Le romantisme est un mouvement de sensibilité et d'idées. dont les premières manifestations se font sentir a la fin du XVIIle siècle et au début du XIXe siécle. II touche une multitude de domaines : histoire, politique, réforme sociale, philosophie, littérature, musique, arts, etce. II véhicule une nouvelle pereeption de la nature, en rupture avec celle du welassicisme du XVIIle siècle. Au XIXe siecle, les paysages naturels sont fortement wattraetifs\%. Les individus cherchent une signification existenticlle à travers la contemplation de la wnaturew. Ce serait cette visée à la fois d'investissement de valeurs dans la nature et de lecture du pay. sage qui semble être la raison profonde de l'émergence des fronts de villégiature dans 
Charlevoix (cf. Gagnon-Prattc, 1980, 1988 : Dubé, 1983, 1985, 1986, 1995).

10 Ligne tracéc par le déplacement du sujet allant d'une position à une autre (Notes du cours GGR-15195), Lat trajectoire n'a de sens que si la position-source et la position-but sont qualitativement differentes. Le sujet va dibrement* de l'une à l'autre en franchissant une discontinuité qui चen vaut la peines. vu que c'est adifférent» de l'autre côté. La phase de mobilité représentée par une trajectoire se défin it selon des attributs de régulation et de directionnalitế. À cet égard, Desmarais et Ritchot specifie que aSi un acteur contrôle son mouvement en direction d'une positions, sa trajectoire est aendoréguleew. Si, a l'inverse, il se dirige vers une position sous la contrainte d'un autre acteur. sạ trajectoire est cexorégulée».. En croisant les modalités d'endorégulation et d'exorégulation atvec les directions (polarisante et diffusante) qui qualifient également les trajectoires, on peut dégager quatre classes de mobilité conduisant à autant de positions distinctes (trajectoires de rassemblement endorégulé foçalisant: de dispersion exorégulée diffusante; de concentration exorégulée focaIsante: d'évasion endoréguléc diffusante) (1997: 44).

11 Traditionnellement. le nomadisme est un genre de vie marginal contraignant des acteurs démunis à de continuels déplacements pour grappiller les ressources indispensables à leur subsistance. La géographie humaine structurale a très tôt insisté sur le fait qu"il existe aussi un nomadisme endorégulé urbain. Le nomadisme urbain est qualifié de *sélectif \%, à la différence du nomadisme effectivement marginal et qualifié pour sa part de «résiduels. Le lecteur intéressề à approfondir davantage la théorie géographique structurale, ici appliquée à l'étude du tourisme en Charlevoix, pourra consulter Ritchot et Mercier, 1992 ; Desmarais, 1995 ; Desmarais et Ritchot, 1997.

\section{BIBLIOGRAPHIE}

Bluteau, Marc-Andre et Serge Gauthier (1987). "Quatre siecles et demi d'histoire au pays de Charlevoix*, Cap-au-Diamants, vol 3, no 3, p. 3-7

Bouchard, Gérard et Lise Bergeron (1989), aAux origines d'une population régionale: mythes et réalitês démographiques et sociales*, Revue d'histoire de l'amerique française, vol 42, no 3, p. $389-409$.

Boyer, Mare (1980), «Evolution sociologique du tourisme: continuite du tourisme rare et rupture contemporaines, Loisir \& Societe, vol 3, no 1, P. $49-82$.

Brière, Roger (1967), *Les grands traits de l'ćvolution du tourisme au Québeco. Bulletin de l'as. sociarion des géographes de l'Amerique fran caise, no 11, p. 83-95.

(1967a), «GÉgraphie du tourisme au Québecs, thèse de doctorat, département de géographie, Université de Montréal.

Bureau, Luc (1977), Des paysages, des idees et des hommes: Ie projet collectif de Charlevoix, Cahiers de gégraphie de Québec, vol 21, no 53 54, p. $187-220$.

Buies, Arthur (1884), Chronique canadienne, hameurs et caprices, Imprimeric de C. Darveau, Québec.

Courville, Serge er al (1988), Seignewries et fiefs du Québec: nonenclaure et cartographie, (coll. Dossiers toponymiques, no 18), Québec, CELAT.

Courville, Serge ed al. (1995), Le pays laurentien au XIX siecle: les morphologies de base-Atlas historigue du Québec, Québec, Les Presses de l'Université Laval.

Courville, Serge el Normand Séguin (1996), Le cô̂t du sol: deur éndes de géographie historiqué Québec, Les Présses de l'Université Laval.

Dechêne, Louise (1968), "Les entreprises de William Price: 1810-1850m, Histoire socialeSocial History, no $1, \mathrm{p}, 16-52$.

(1977), william Price». in Dictionaire biographique du Canada, Volume IX de 1861 à 1870, Toronto et Quebec, University of Toronto Press et Les Presses de I'Universite Laval, p. 704-708.

Des Gagniers, Jean (1994), Charlevoix, pays enchante, Québec, Les Presses de I"Université Laval.

Desmarais, Gaëtan (1995). La morphogenèse de Paris, des origines à la révolution, Parisł Québec, L'HarmattanไCELAT

Desmarais, Gacetan et Gilles Ritchot (1997), «La dimension morphodynamique des grands établissements humains : l'exemple de Montréals, Visio, woll 2 , $\pi 02$, p. $43-5$ ?

Dube, Philippe (1983). "Faire l'histoire du pays

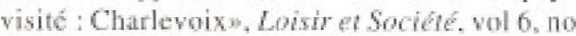
1, p. $211-228$

(1985), wCharlevoix : nos châteaux de la Loirex, Continuile, p.34-35.

(1986), Dear cents ans de villégiature dans Charlevoix. Lhistoire d'un pays visite. Quebec, Les Presses de l'Université Laval.

(1995), «La villégiature dans Charlevoix: une tradition séculaire, un patrimoine encore vivantw, Teoros, vol 14, no 2, p. 4-7.

Gagnon, France (1992), «Du cheval au rail: l'évolution des circuits touristiques québecois au XIX siecles. In Le pays lauremien au XIXe siècle, Cahier 7, Université Laval, Université du Québec à Montréal, Université du Québec à Trois-Rivières, p. 101-133.

Gagnon, Gaston (1988), Un pays neuf; Le Saguenay-Lac-Sant-Jean én évolution, Alma, Les Editions du Royaume.
Gagnon, Serge (1996), *L'émergence du tourisme atu XIX sicele : l'exemple de Charlevoix, une analyse morphologique, dynamique et sémiotique», mémoire de maîtrise, département de géographie, Université Laval.

Gagion-Pratte, France (1980), L'anchitecture de la nature à Québec au dix-neuvième siecle : les villas.

Gagnon-Pratte, France et Philippe Dubé (1988), La villa : ou l'art de vivre en harmonie avec la natures, Continuite, no 40, p. 22-25

Gauthicr, Serge (1989), wa Malbaie», Continuire, no 44, p. 48-55.

Lalancette, Mario (1987), aAlexis Tremblay Picote (1787-1859)\%, Revue Charlevoix, Société d'histoire de Charlevoix, voll 2, no 1, p. 9-12.

Lapointe, Adam, Paul Prévost et Jean-Paul Simard (1981), Economie regionale da Saguenay-Lac-Saint-Jean. Montrẹal, Gaětan Morin Editcur.

Larocque. Paul (1993), Parcours historique dans la region touristique du Bas-Sant-Laurent, Rimouski, GRIDEQ

Ouellet, Fernand (1978), wPropriété seigneuriale et groupes sociaux dans la vallé du Saint-Laurents, in Melanges d'histoire du Canada francais offers a professeur Mancel Trudel, Cahiers du centre de recherche en civilisation canadiennefrançaise, no 14, Ottawit, Édition de l'Univer=sité d'Ottawa, p 183-213.

Paradis. Alexandre (1948), Kamouraska (/6741948), Québec.

Raveneau, Jean (1977), analyse morphologique, classification el protection des paysages: le cas de Charlevoix., Cahiers de géographe de Quebec, vol 21, no 53-54, p. 135-186.

Ritchot, Gilles et Claude Felta (1985). Forme urbaine et pratique sociale, (coll. Science et Thẻorie), Montréal, éd, Le Préambule.

Ritchot, Gilles (1991). Eludes de géographie structurale, (coll. Cahiers spéciaux, no 15). Québec, Centre de recherche en aménagement et développement.

Ritchot, Gilles et Guy Mercier (1992), «Numéro spécial; La géographie humaine structurales, Cahiers de géographie du Ouebec. Québec. Université Laval, vol 36. no 98, p. 167-336.

Saint-Amour, Jean-Pierte (1979), La villégiamre au Quebec, problénatique de l'aménagement da territoire, Hull, Edition Asticou.

Simard, Leo (1987), La petite histoire de Charlevoir, Club Lions de Clermont-La MalbaiePointe-au-Pic Inc.

Trépanièr, Paul (1988), «Un hêritage romantiquew, Continuite, no 40, p. 5.

Vézina, Raymond (1977), «L'art documentaire au service des sciences humaines : le cas du comté de Charlevoix au Québecs, Cahiers de geographie de Québec, vol 21, no 53-54, p. 293308. 